\title{
OpenVape: An Open-Source E-Cigarette Vapor Exposure Device for Rodents
}

\author{
Jude A. Frie, ${ }^{1}$ Jacob Underhill, ${ }^{1}$ Bin Zhao, ${ }^{2}$ Giordano de Guglielmo, ${ }^{3}$ Rachel F. Tyndale, ${ }^{4}$ and \\ (1)Jibran Y. Khokhar'
}

\section{https://doi.org/10.1523/ENEURO.0279-20.2020}

${ }^{1}$ Department of Biomedical Sciences, Ontario Veterinary College, University of Guelph, Guelph, Ontario, Canada, N1G 2W1, ${ }^{2}$ Departments of Pharmacology and Toxicology, University of Toronto, Toronto, Ontario, Canada, M5S 1A8, ${ }^{3}$ Department of Psychiatry, University of California, San Diego, San Diego, CA, 94720, and ${ }^{4}$ Departments of Pharmacology and Toxicology, and Psychiatry, University of Toronto, Ontario Canada, M5S 1A8, Centre for Addiction and Mental Health, Toronto, Ontario, Canada M6J $1 \mathrm{H} 4$

\section{Visual Abstract}

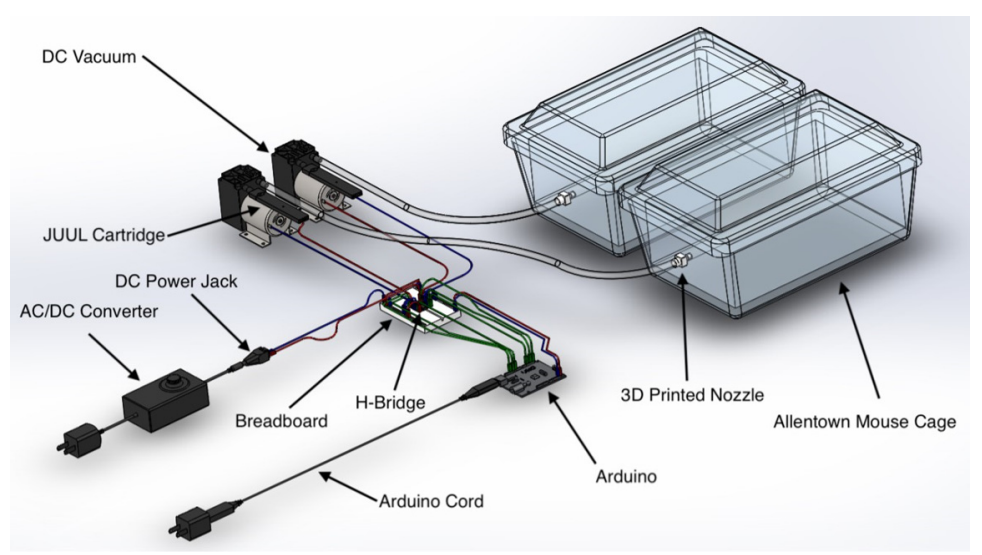

The prevalence of "vaping" has recently seen significant increases in North America, especially in adolescents. However, the behavioral correlates of vaping are largely unexplored. The uptake of existing technologies meant for rodent vapor inhalation remains limited because of a lack of affordability and versatility (ability to be used with a variety of vaporizers). The OpenVape (OV) offers an open-source, low-cost solution that can be used in a variety of research contexts. Here, we present a specific use case, combining the OV apparatus with JUUL e-cigarettes. This apparatus consists of Arduino-operated vacuum pumps that deliver vapor directly from e-cigarettes to exposure chambers. The OV is easy to build and customize for any type of vaporizer

\section{Significance Statement}

With the recent increases in popularity of vaping, behavioral and neurobiological studies in preclinical models will be pivotal in exploring the impacts of this use. While there are commercially available vapor exposure equipment, they can be prohibitively expensive and often require proprietary software. The OpenVape (OV) is able to deliver regulated doses of vapor into a standard animal cage with minimal operator intervention. The OV is also open-source, easy to build, inexpensive, and can be used in a variety of research contexts because of its small physical footprint. In this study, we validate its efficacy using JUUL e-cigarettes, showing that animals achieve meaningful nicotine levels, and that both adolescent and adult animals display conditioned place preference (CPP) to the nicotine vapor. 
(e.g., nicotine pod or tank; cannabis flower or concentrates). To test the OV, we performed biochemical verification and behavioral studies. The behavioral test (conditioned place preference, CPP) was conducted using adolescent and adult animals to assess developmental differences in the rewarding effects of nicotine vapor, as previously observed with injected nicotine. These findings demonstrate that even after brief exposures to nicotine vapor, pharmacologically relevant nicotine and cotinine levels could be detected in plasma, and significant CPP was observed, especially in adolescent rats which showed preference at shorter puff delivery durations (lower nicotine doses) compared with adults. Together, these findings suggest that OV provides an affordable, open-source option for preclinical behavioral research into the effects of vaping.

Key words: adolescent; e-cigarette; JUUL; nicotine; reward; vaping

\section{Introduction}

In recent years, adolescent e-cigarette use has increased dramatically, with past $30-d$ nicotine vaping among United States high school students growing from $11.7 \%$ to $27.5 \%$ between 2017 and 2019 (Wang et al., 2019). Currently, the most popular e-cigarette is the JUUL, maintaining $76 \%$ of the United States e-cigarette market (Craver, 2019). JUUL's adolescent appeal has been attributed to their sleek/discreet design (Kavuluru et al., 2019), influential marketing (Mantey et al., 2016; Huang et al., 2019), appealing flavors (Leavens et al., 2019), and high nicotine content (Kong et al., 2019). Additionally, JUULs have been shown to deliver significantly more nicotine to the bloodstream compared with traditional cigarettes (Rao et al., 2020).

Adolescence is thought to be a period of increased sensitivity to the rewarding effects of nicotine (Thorpe et al., 2020). Indeed, nearly $90 \%$ of cigarette smokers initiate smoking before the age of 18 (Substance Abuse and Mental Health Services Administration, 2013). Adolescent smokers also increase cigarette intake faster than adults (DiFranza et al., 2000) and report more positive and less aversive experiences to initial cigarette exposures (Benowitz, 1999) Animal studies support this data, with adolescent animals consistently showing both greater preference, and preference across a larger range of doses during nicotine conditioned place preference (CPP) compared with

Received June 27, 2020; accepted August 18, 2020; First published August 28, 2020.

R.F.T. has consulted for Quinn Emanuel and Ethismos Research Inc on unrelated topics. All other authors declare no competing financial interests.

Author contributions: J.A.F., J.U., G.d.G., and J.Y.K. designed research; J.A.F., J.U., B.Z., R.F.T., and J.Y.K. performed research; J.A.F., J.U., and J.Y.K. contributed unpublished reagents/analytic tools; J.A.F., J.U., and J.Y.K. analyzed data; J.A.F., J.U., R.F.T., and J.Y.K. wrote the paper.

This work was supported by the Natural Sciences and Engineering Research Council Discovery Grant RGPIN-2019-05121 and Canadian Institutes of Health Research Catalyst Grant 442011 (to J.Y.K.). This work was also supported, in part, by funding from the Canada Research Chairs Program (R.F. T., CRC in Pharmacogenomics) and the Canadian Institutes of Health Research Foundation Grant FDN-154294.

Acknowledgements: We thank Judy Chen and Ariel Oren for their assistance with CPP experiments.

Correspondence should be addressed to Jibran Y. Khokhar at jkhokhar@ uoguelph.ca.

https://doi.org/10.1523/ENEURO.0279-20.2020

Copyright @ 2020 Frie et al.

This is an open-access article distributed under the terms of the Creative Commons Attribution 4.0 International license, which permits unrestricted use, distribution and reproduction in any medium provided that the original work is properly attributed. adults, a measure of the reward-like (hedonic value) properties of a drug (Vastola et al., 2002; Belluzzi et al., 2004; Shram et al., 2006; Brielmaier et al., 2007; Kota et al., 2007, 2008; Torres et al., 2008; Shram and Lê, 2010; Ahsan et al., 2014). While beneficial in understanding the rewarding effects of nicotine, these studies may not capture what makes nicotine vapor rewarding in adolescents. The current study improves on the translatability of findings by directly exposing rats to the most popular e-cigarette available, JUUL. No studies to date have assessed the developmental differences in nicotine vapor preference, partly owing to limited options for exposing rodents to nicotine vapor.

Current commercially available nicotine vapor exposure apparatuses (e.g., DSI Buxco, SCIREQ inExpose, LJARI eVape) are prohibitively expensive and require proprietary hardware and software to operate, thereby limiting their accessibility and versatility. This has led others to create customized alternatives (Manwell et al., 2014; Liu et al., 2016; Nguyen et al., 2016; Hage et al., 2017; Lefever et al., 2017; Hilpert et al., 2019). However, these alternatives are still very expensive, complicated to construct, and are not always open-source. Thus, to address these issues, we created OpenVape (OV), an affordable, open-source vapor delivery system. OV can be made for $\sim \$ 230$, is easily constructed/operated, and is highly versatile (can be used with a variety of vaping devices and products). It is also open-source, encouraging the community to continue to add functionality as required. Here, we provide the instructions to build the simplest version of OV and provide a relevant behavioral validation showing the developmental differences in nicotine's reward-like properties during a CPP task. We also show that OV is capable of producing pharmacologically relevant blood nicotine and cotinine levels following short periods of vapor exposure.

\section{Materials and Methods}

\section{Animals}

For the pharmacokinetic study, 15 adult male Sprague (Charles River) were used. These rats were housed in pairs and given ad libitum access to standard chow and water. For the age-dependent CPP study, a total of 48 male Sprague Dawley rats were used, consisting of 24 adults and 24 adolescents. These rats were housed in groups of four (to maintain consistency between adult and adolescent rats) and given access to standard chow and water. All animal procedures were performed in accordance with the [University of Guelph] animal care committee's regulations. 
Table 1: Bill of materials

\begin{tabular}{|c|c|c|c|}
\hline Component & Quantity & Price & Source of materials \\
\hline Arduino Uno (with cable) & 1 & $\$ 12.99$ & www.amazon.ca \\
\hline H-bridge motor controller & 1 & $\$ 1.89$ & www.amazon.ca \\
\hline Solderless breadboard & 1 & $\$ 3.49$ & www.amazon.ca \\
\hline AC/DC converter (with power jack) & 1 & $\$ 13.49$ & www.amazon.ca \\
\hline Jumper cables & 120 & $\$ 6.98$ & www.amazon.ca \\
\hline Allentown mouse cages & 2 & - & In-Lab \\
\hline 3D-printed nozzles & 2 & - & In-Lab \\
\hline PCB (optional), replaces H-bridge, breadboard, and jumper cables & 1 & - & - \\
\hline Total & $\$ 230.88(C A D)$ & & \\
\hline
\end{tabular}

\section{Device design}

The OV device requires one standard wall receptacle for power. OV is compact and does not require extensive lab space to store or for use. The device can be built for $\sim \$ 230 \mathrm{CAD}$ in parts per apparatus (Table 1). The simple construction and programming of this design also does not require extensive coding or electronic/circuits experience. The components required to build the OV, and the step-by-step instructions are presented below.

An Arduino microcontroller takes simple instructions from code and relays them to an $\mathrm{H}$-bridge, which sends the required signals to the motors.

\section{Build instructions}

Detailed step-by-step OV building instructions, Arduino code, 3D printer files, and electrical layouts are all available at https://www.khokharlab.com/open-source-file-downloads. A brief overview of the instructions is included in Table 2 (for clarification, see Figs. 1, 2 and Movie 1).

A custom printed circuit board (PCB) has also been included. This simplifies the construction of the device as it eliminates the need for many wired connections. The small PCB is plugged into the Arduino to control all logic commands. The board replaces the $\mathrm{H}$-bridge, breadboard, and many wired connections while increasing simplicity of construction and reducing cost even further. For ease of replication the design has been included below in Figure 3.

\section{Code accessibility}

The code/software described in the article is freely available online at https://www.khokharlab.com/opensource-file-downloads. The code is available as Extended Data 1.

\section{Operating instructions}

To customize the interval time, the uploaded Arduino code must be adjusted. In the brackets after "DELAY" the number of seconds $(1000 \times)$ of vapor administration required can be entered, followed by the seconds $(1000 \times)$ of rest required. The looped code will repeat this sequence forever. However, a time constraint can be set if needed. The default code pulses on for $2 \mathrm{~s}$, then rests for $4 \mathrm{~s}$. These parameters were determined through iterative experimentation to be the optimum timing to maximize the amount of vapor produced, while not overheating the JUUL device; these parameters can be modified to optimize vapor delivery from other vaporizers.

After uploading the desired code, the system can be turned on by rotating the voltage dial to $6 \mathrm{~V}$. Similar to the

Table 2: Build instructions

\begin{tabular}{ll}
\hline Step & Instructions \\
\hline 1 & Download the code file from https://www.khokharlab.com/open-source-file-downloads \\
2 & Print the nozzles from https://www.khokharlab.com/open-source-file-downloads using a 3D printer \\
3 & Attach the H-bridge to the breadboard \\
4 & Wire the Arduino to the system with six "logic" cables, a power cable, and a ground \\
5 & Connect the two terminals of each motor to the corresponding H-bridge pins (Fig. 2) \\
6 & Insert two cables into the DC power jack (one for ground and one to power the motors) \\
7 & Secure a charged and filled JUUL e-cigarette to each of the two motors with heat shrink \\
8 & On the "out" ports of each motor, tightly heat shrink one end of an 8mm plastic tubes onto it \\
9 & Heat shrink the 3D-printed nozzles onto the opposite ends of each 8-mm plastic tube and insert the nozzles \\
10 & into the drilled holes on the ends of each of the Allentown mouse cages \\
11 & Plug the Arduino board into your computer and using the Arduino IDE, upload the appropriate code onto the board \\
12 & Plug both the Arduino and the adjustable voltage supply into a receptacle. The cable to the Arduino will automatically \\
& supply the desired voltage to the board \\
& Adjust the voltage supply to 6 V, and vapor clouds will appear at the specified intervals
\end{tabular}




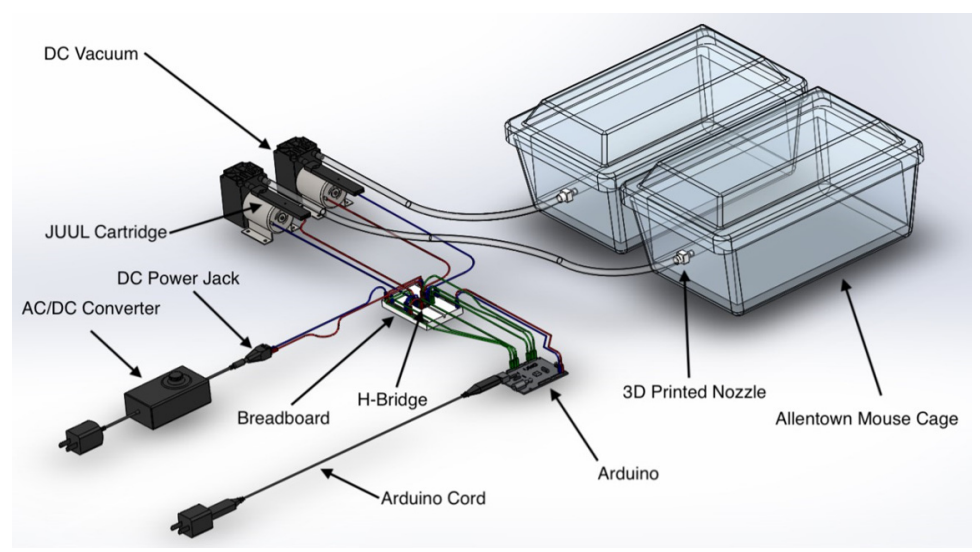

Figure 1. Labeled schematic of the OV system.

timing parameters, this voltage setting was found to dispense the optimal vapor clouds (as seen in Movie 2) while ensuring the JUUL does not overheat or melt the pod. The system can be turned off by returning the dial to $0 \mathrm{~V}$. This setting can also be adjusted based on the vaporizer used through an iterative process.

\section{JUUL-specific instructions}

For the specific use case with JUUL, the JUUL e-cigarette's battery life (200 inhales) is large enough for most applications but will occasionally require recharging. The battery on the JUUL indicates whether the power is running low by flashing red. The battery can be charged by

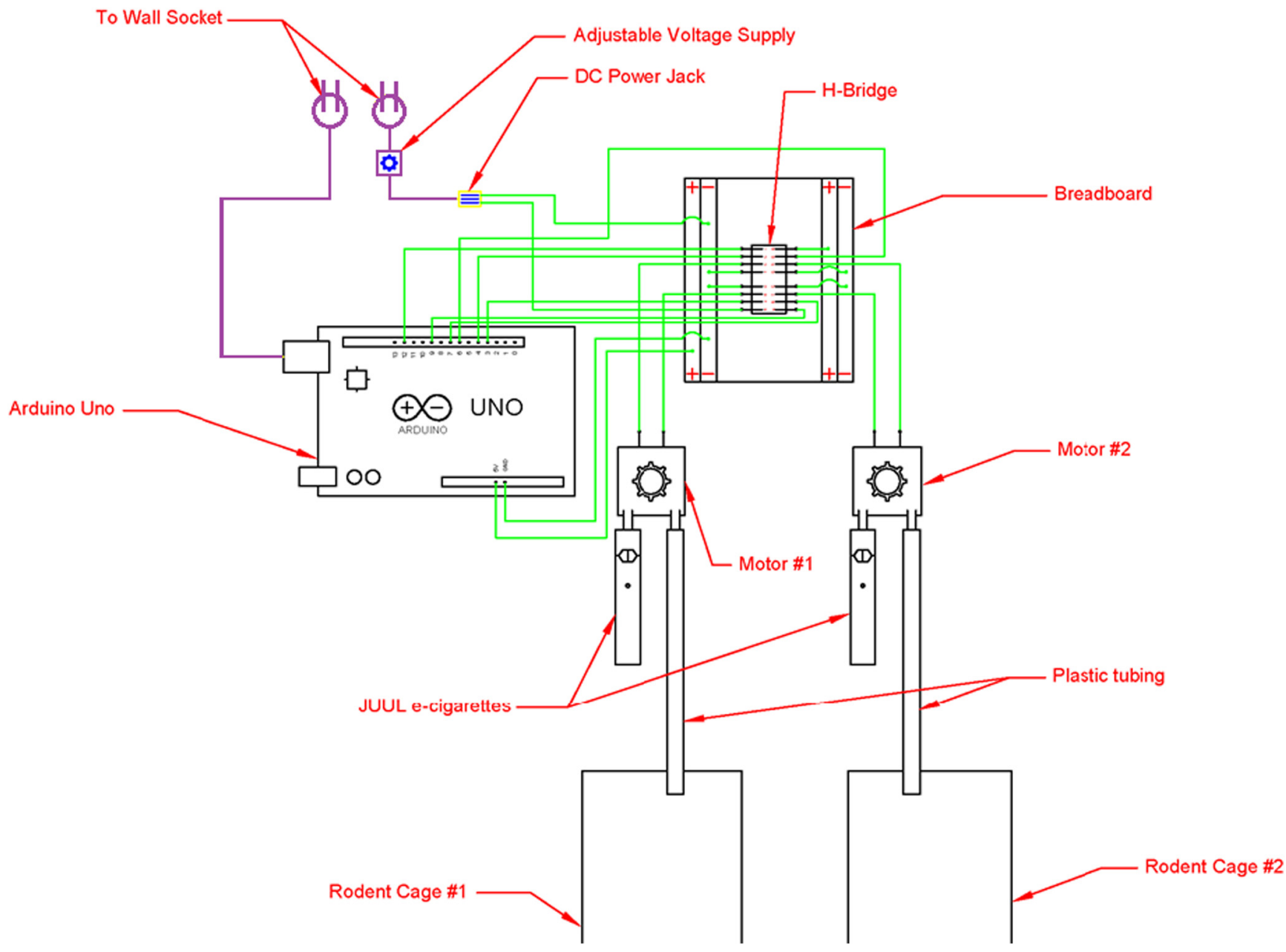

Figure 2. Wiring diagram for OV apparatus. 


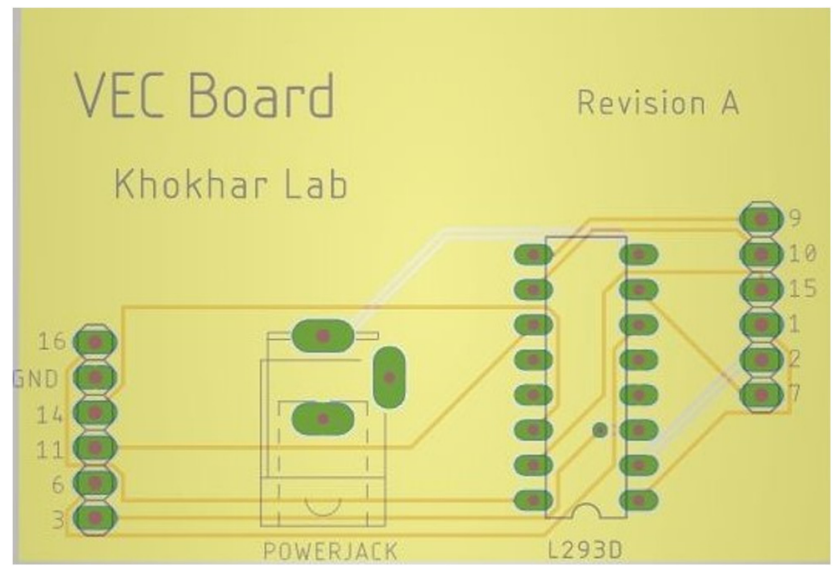

Figure 3. Custom $\mathrm{PCB}$ design to facilitate building the $\mathrm{OV}$ apparatus.

separating the JUUL from the heat shrink and placing the JUUL upright on the provided USB charger until the LED glows green. The other maintenance task is replacing the JUUL pod. Conveniently, the battery lasts approximately as long as the JUUL pod does, therefore, if the code provided is used, the system can run at least 20 min without the need for recharging. The pods can be replaced with a new pod or refilled for the vehicle groups. The vehicle pod can be refilled by gently removing the lid of the pod and injecting the e-liquid vehicle into the pod with a syringe.

\section{Drugs}

JUUL mango $5 \%$ nicotine e-liquid $(59 \mathrm{mg} / \mathrm{ml})$ or vehicle eliquid was administered. Vehicle e-liquid was mixed based on a recent gas chromatography-mass spectrometry study that established the primary JUUL mango flavor constituents (ethyl maltol, 3-hexen-1-ol, ethyl butanoate, and $\delta$-undecalactone; Advanced Biotech), and a 30:60 mixture of propylene glycol and glycerol (Omaiye et al., 2019). The dose of nicotine is determined by the duration of time that the motor is running and pumping vapor into the cage (including puffs and timeouts).

\section{Plasma nicotine and cotinine concentrations}

Adult male rats $(N=15$; distinct from animals used for CPP) were divided into groups of five, each group receiving a different dose. Each group received 2, 4, or $8 \mathrm{~min}$ of pump activation; thereby, altering the amount of vapor in the cage. Each epoch of pump activation produced a 2-s puff duration, followed by a 4-s time out resulting in 10 2-s puffs/min (e.g., 2-min group received 20 2-s-long puffs). Although the device only produced vapor for 2, 4, or $8 \mathrm{~min}$, all animals spent a total of $10 \mathrm{~min}$ in the chambers. Following the 2, 4, or 8 min of pump activation (including puffs and time-outs), the device is turned off and the rat is allowed to remain in the chamber for the remaining time $(8,6$, and $2 \mathrm{~min}$, respectively). Thus, the time in the chamber was always $10 \mathrm{~min}$, but the vapor concentration differed based on the duration of pump activation. After one session of their respective vapor dose, saphenous blood draws were conducted for each animal 10 and $120 \mathrm{~min}$ after the end of exposure $(20$ and $130 \mathrm{~min}$ after exposure initiation). The collected blood samples were centrifuged at $1400 \times g$ for $10 \mathrm{~min}$. The resulting plasma was collected and stored at $-4^{\circ} \mathrm{C}$. Liquid chromatography with tandem mass spectrometry (LC-MS/MS) analysis was used to quantify the levels of nicotine and cotinine following previously published sample preparation and quantification methods (Craig et al., 2014). The plasma levels were only assessed in adult male rats as a validation of the efficacy of the device in delivering pharmacologically relevant doses of nicotine; the impact of age and sex on nicotine vapor pharmacokinetics will be addressed in a more comprehensive manner in upcoming studies from our group.

\section{CPP}

\section{Dosing for CPP}

The group of animals was divided by age (24 adult and 24 adolescent) then each age group was further divided into four unique dosage groups. The first group was a vehicle control and was exposed to the vehicle vapor on every conditioning day with a dose of $4 \mathrm{~min}$ (as a control for the biased CPP design used in this article). The remaining groups received a dose of 2,4 , or 8 min depending on the group. Whether the vapor contained nicotine or vehicle solution depended on the day of the study. Cage mates were exposed together to the vapor to avoid isolation effects but were conditioned in the CPP apparatus individually in a biased design.

\section{Apparatus}

The CPP apparatus has two chambers separated by a wall with a guillotine door (Shuttle Boxes from Coulbourn Instruments). Each chamber $(30 \mathrm{~cm}$ wide and $30 \mathrm{~cm}$ long) has Plexiglas surrounding walls that stand $30 \mathrm{~cm}$ high. A large, clear sheet of Plexiglas is placed over top of the chambers. Both chambers have unique visual, tactile and olfactory cues. Chamber 1 has polka dotted walls, barred floors, and a vanilla scent. Chamber 2 has pinstriped walls, grated floors, and a lemon scent. An Ethovision XT system (Noldus) was used to record the amount of time the animals spent in each chamber.

A biased CPP protocol was chosen as it has been suggested that this protocol is better suited to assess nicotine CPP; small preferences for one chamber have been shown to play a significant role in nicotine CPP development (Clarke and Fibiger, 1987; Shoaib et al., 1994; Le Foll and Goldberg, 2005). The CPP procedure consists of three phases: habituation, conditioning, and postconditioning. The protocol used for CPP is described as seen in Figure 4.

\section{Days 1-3: habituation}

During days 1 and 2 of habituation, the guillotine door is raised, and the animals roam freely through both chambers for $15 \mathrm{~min}$. On the third day, throughout the 15-min session, the time animals spend on each side of the chamber is recorded as a baseline preference.

\section{Days 4-9: conditioning}

During the conditioning phase, the guillotine door is closed, and the animals are restricted to a specific side of 


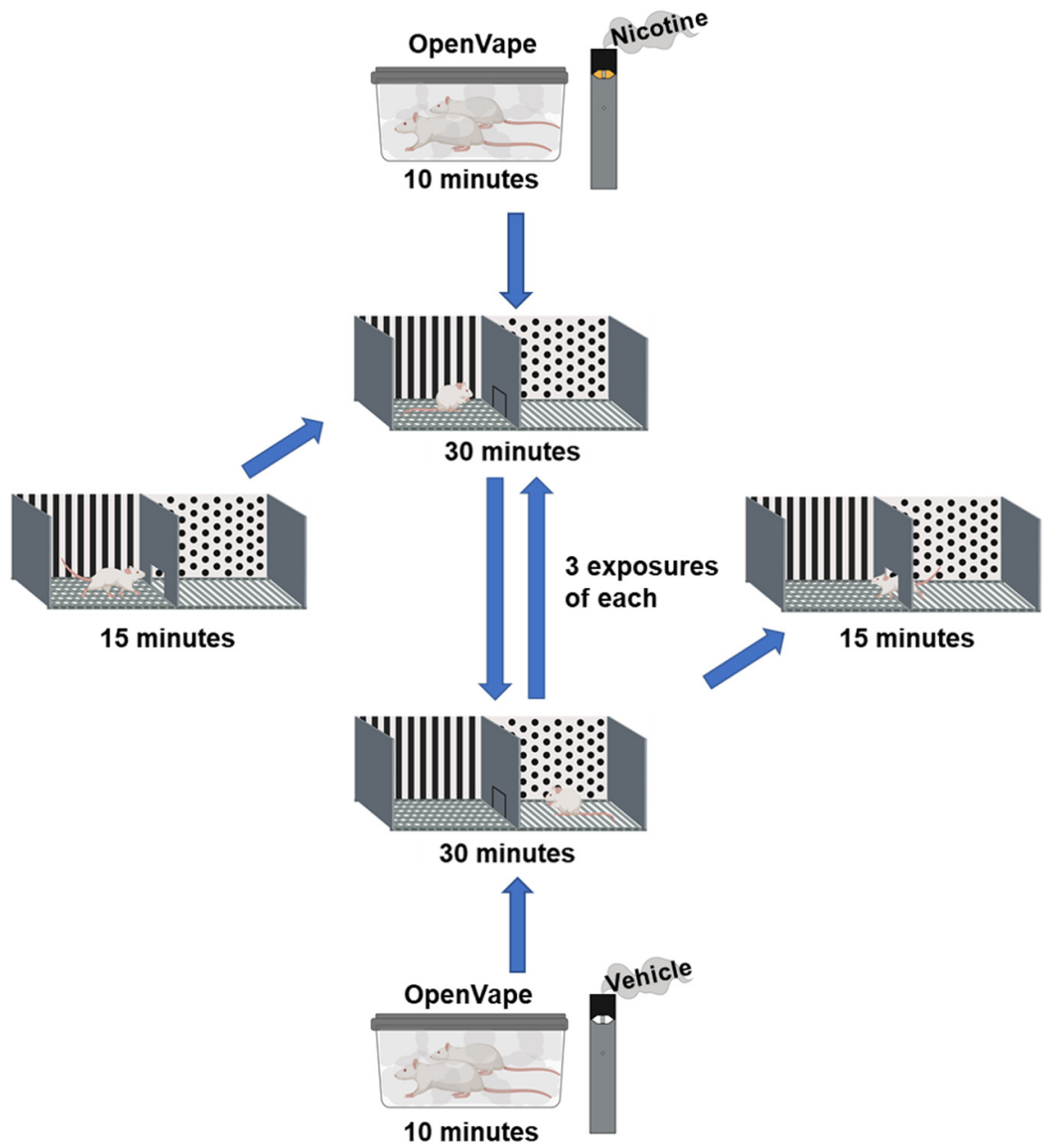

Figure 4. Visual schematic outlining the CPP paradigm. Figure made using BioRender.

the apparatus. After being removed from their home cages and exposed to JUUL nicotine vapor for $10 \mathrm{~min}$ in the OV vapor exposure chambers, animals were then individually confined to their initially non-preferred chamber of the CPP apparatus (on nicotine days) for $30 \mathrm{~min}$. Following the 10-min exposure to the vehicle solution vapor, the animals were confined to their initially preferred chamber (on vehicle exposure days). The rats receive these treatments on alternating days with the nicotine/vehicle start day counterbalanced across animals. The vehicle control animals received vehicle vapor on all days and were confined on alternating days to the preferred and non-preferred chambers.

\section{Day 10: postconditioning}

During the postconditioning phase, no vapor was administered and the rats were again allowed access to both chambers with the guillotine door open for $15 \mathrm{~min}$. The time each animal spent in each chamber was recorded to assess each animal's final preference.

\section{Statistical analysis}

Statistics were conducted using IBM SPSS Statistics 25 and GraphPad Prism 6.0 with a random (superiority) design.
For CPP, a conditioning index was calculated as the difference in time spend in the initially preferred chamber preconditioning to postconditioning. Outliers were defined as animals whose difference in time on the drug paired side was $>2$ SDs from the mean. Three of the adolescent rats were deemed outliers for the CPP study and were not included in the analysis, thus a total of 45 animals were analyzed. Overall comparisons were evaluated using a two-way ANOVA (treatment $\times$ age or treatment $\times$ sex). The determination of whether CPP was established was based on Bonferroni corrected one-sample $t$ tests compared with a theoretical conditioning index of $0 ; \alpha$ was initially set at 0.05 and was adjusted down via Bonferroni correction to account for the number of comparisons such that results were only significant if $p<0.0125$. For plasma nicotine and cotinine, a two-way (dose $\times$ time) ANOVA was conducted followed by trend analysis for each time point.

\section{Results}

\section{Dose-dependent plasma cotinine levels observed in adult rats exposed to nicotine vapor}

The results from the plasma nicotine concentration assessment are shown in Figure 5. Plasma nicotine levels of 

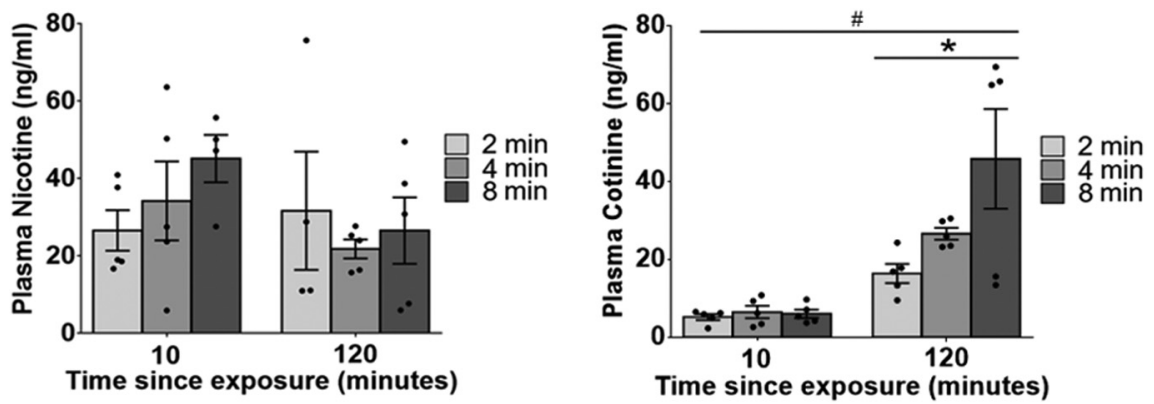

Figure 5. Plasma concentrations for nicotine (left) and cotinine (right) at 10 or 120 min after termination of a 10-min total exposure session during which vapor was delivered for one of 2, 4, or 8 min. Data represented as mean \pm standard error of the mean (SEM); $\# p<0.0001120$ - versus 10-min time point; $* p<0.05$ dose trend.

$26.6,34.2$, and $45.2 \mathrm{ng} / \mathrm{ml}$ were observed $10 \mathrm{~min}$ after 2-, $4-$, and 8-min nicotine vapor exposure doses, respectively. Nicotine could still be detected in the plasma 120 min after exposure. Two-way ANOVA revealed no significant effects of time $\left(F_{(1,12)}=1.626, p=0.226^{a}, \eta_{p}^{2}=\right.$ 0.119 ; Table 3) or dose $\left(F_{(2,12)}=0.649, p=0.540^{\mathrm{b}}, \eta_{\mathrm{p}}^{2}=\right.$ 0.098 ; Table 3 ) on plasma nicotine levels and no interaction was observed $\left(F_{(2,12)}=1.093, p=0.366^{\mathrm{c}}, \eta_{\mathrm{p}}^{2}=0.154\right.$; Table 3).

As expected, plasma cotinine levels were low at $10 \mathrm{~min}$ after removal from OV chambers; an increase in cotinine levels, which was dose-related, was seen 120 min after nicotine vapor exposure. Two-way ANOVA revealed a significant effect of time $\left(F_{(1,12)}=29.78, p=0.0001^{\mathrm{d}}, \eta_{\mathrm{p}}^{2}=0.713\right.$; Table 3$)$ but not dose $\left(F_{(2,12)}=3.81, p=0.0523^{\mathrm{e}}, \eta_{\mathrm{p}}^{2}=0.389\right.$; Table 3$)$ on plasma cotinine levels. There was also no interaction between dose and time $\left(F_{(2,12)}=3.782, p=0.0533^{f}, \eta_{p}^{2}=0.387\right.$; Table 3). Trend analysis revealed a linear dose trend in plasma cotinine levels $120 \mathrm{~min}$ after exposure $\left(p=0.018^{\mathrm{g}}, R^{2}=\right.$ 0.3812, $\eta_{p}^{2}=0.386$; Table 3).

\section{Developmental differences in CPP for nicotine vapor}

The results of the age-dependent CPP experiment are shown in Figure 6. Significant CPP was observed in adult

Table 3: Statistical table

\begin{tabular}{llll}
\hline & Data structure & Type of test & Power \\
\hline $\mathrm{a}$ & Normal & Two-way ANOVA & 0.217 \\
$\mathrm{~b}$ & Normal & Two-way ANOVA & 0.134 \\
$\mathrm{c}$ & Normal & Two-way ANOVA & 0.198 \\
$\mathrm{~d}$ & Normal & Two-way ANOVA & 0.999 \\
$\mathrm{e}$ & Normal & Two-way ANOVA & 0.577 \\
$\mathrm{f}$ & Normal & Two-way ANOVA & 0.573 \\
$\mathrm{~g}$ & Normal & Linear contrast & 0.713 \\
$\mathrm{~h}$ & Normal & One-sample $t$ test & 0.866 \\
$\mathrm{i}$ & Normal & One-sample $t$ test & 0.996 \\
$\mathrm{j}$ & Normal & One-sample $t$ test & 1.000 \\
$\mathrm{k}$ & Normal & Two-way ANOVA & 0.864 \\
$\mathrm{l}$ & Normal & Two-way ANOVA & 0.969 \\
$\mathrm{~m}$ & Normal & Two-way ANOVA & 0.337 \\
$\mathrm{n}$ & Normal & Linear contrast & 0.935 \\
$\mathrm{o}$ & Normal & One-sample $t$ test & 0.956 \\
$\mathrm{p}$ & Normal & Two-way ANOVA & 0.777 \\
$\mathrm{q}$ & Normal & Two-way ANOVA & 0.176 \\
$\mathrm{r}$ & Normal & Two-way ANOVA & 0.051 \\
\hline
\end{tabular}

rats exposed to $8 \mathrm{~min}$ of nicotine vapor $\left(t_{(5)}=-3.86\right.$, $p<0.006 \mathrm{~h}, \eta_{\mathrm{p}}^{2}=$ Cohen's $d=1.578$; Table 3 ), whereas adolescent rats displayed significant place preference at both the $4 \min \left(t_{(4)}=-6.41, p<0.0022^{\mathrm{i}}\right.$, Cohen's $d=2.865$; Table 3) and $8 \mathrm{~min}\left(t_{(5)}=-13.73, p<0.00002^{\mathrm{j}}\right.$, Cohen's $d=5.606$; Table 3) exposure durations. No change in place preference was observed in the vehicle exposed groups. Two-way ANOVA revealed a significant effect of age $\left(F_{(1,37)}=9.872, p<0.003^{\mathrm{k}}, \eta_{\mathrm{p}}^{2}=0.211\right.$; Table 3$)$ and dose $\left(F_{(3,37)}=7.098, p<0.001{ }^{1}, \eta_{\mathrm{p}}^{2}=0.365\right.$; Table 3$)$ but no interaction $\left(F_{(3,37)}=1.384, p<0.263^{\mathrm{m}}, \eta_{\mathrm{p}}^{2}=0.101\right.$; Table 3). Adolescents also showed a significant linear dose trend $\left(p<0.002^{n}, R^{2}=0.4211, \eta_{p}^{2}=0.445\right.$; Table 3).

\section{Discussion}

This article describes the design of an open-source e-cigarette vapor exposure device for use with rodents. The device was tested with commercially available JUUL e-cigarettes and found to produce pharmacologically relevant plasma nicotine and cotinine levels, as well as behavioral effects in a CPP paradigm. The results of the present study provide the first evidence that brief JUUL e-cigarette vapor exposures are enough to produce CPP, and that similar to other routes of nicotine administration, nicotine vapor may have stronger reward-like properties when administered in adolescence. Although it is difficult to compare CPP results between studies because of methodological differences, the change in preference of our highest dose duration (8-min puff exposure) appears to be roughly equivalent with those found with subcutaneous injections of $0.6 \mathrm{mg} / \mathrm{kg}$ (Torres et al., 2008). This is consistent with the plasma nicotine levels observed at the longest exposure duration, with levels roughly equivalent to the peak concentrations seen with subcutaneous injections of $0.5 \mathrm{mg} / \mathrm{kg}$ (Lefever et al., 2017). These levels are also akin to those seen in adult cigarette smokers (Matta et al., 2007). The present results help validate OV as a reliable behavioral neuroscience tool for use in addiction research, highlighting its utility despite the device's low cost and simple design.

Our behavioral findings are largely consistent with previous age-related studies on nicotine CPP. Overall, the enhancement of nicotine CPP in adolescent rodents has been observed across multiple studies, even with significant differences in methodology (Vastola et al., 2002; 

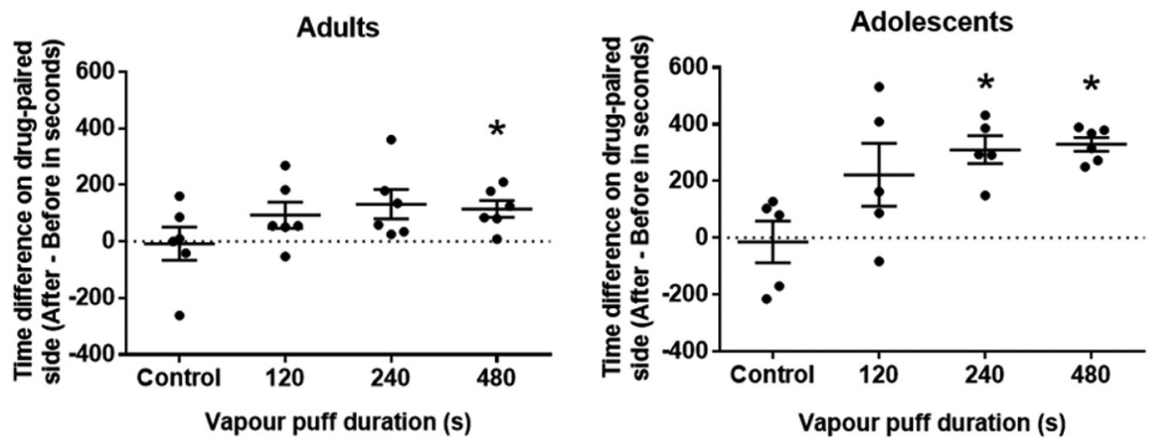

Figure 6. Nicotine vapor CPP in adult and adolescent (PND30-PND39) rats; $* p<0.0125$ postconditioned versus preconditioned (Bonferroni corrected). Data represented as mean \pm SEM.

Belluzzi et al., 2004; Shram et al., 2006; Brielmaier et al., 2007; Kota et al., 2007, 2008; Torres et al., 2008; Shram and Lê, 2010; Ahsan et al., 2014). The specifics of these enhancements, however, are not always consistent, likely owing to these methodological differences. These discrepancies include the time animals spend in the conditioning chamber, number of conditioning days, time between exposures, dosage, method of habituation, age of exposures, route of administration, species, strain, statistical methods, chamber type (two-chamber vs threechamber), and chamber conditions (visual, tactile, and olfactory cues). The resulting differences in observed nicotine CPP enhancements range from whether adolescent rodents permit nicotine reward (Belluzzi et al., 2004; Brielmaier et al., 2007; Shram et al., 2006; Vastola et al., 2002), find nicotine rewarding at lower doses (Ahsan et al., 2014; Kota et al., 2007, 2009), or find nicotine rewarding at higher doses compared with adults (Torres et al., 2008). Our results are most consistent with the finding that adolescents find nicotine rewarding at lower doses compared with adults, although our dose never reached the point at which nicotine becomes aversive; therefore, it is unknown whether the adolescent rats would have also found higher doses less aversive rewarding than adult rats.

In the past, aerosol exposures have required commercial devices with a large footprint that were costly, complicated to operate, and incompatible with the newest forms of vaping devices (Alasmari et al., 2018). To avoid these issues, some labs have employed rudimentary exposure regimens such as placing animals into chambers above

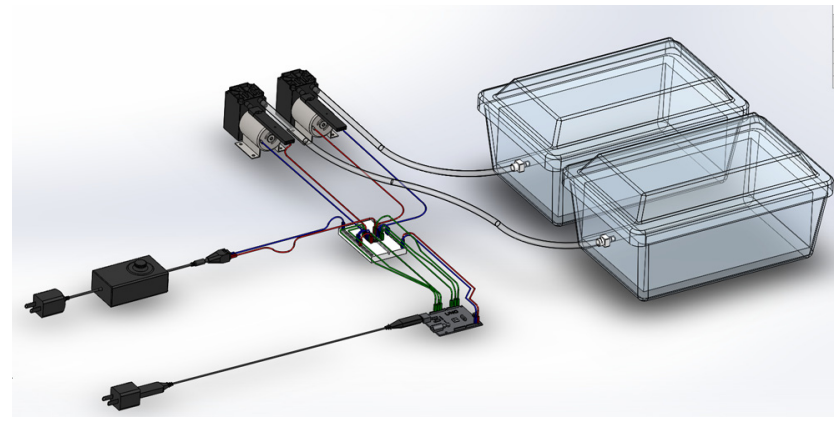

Movie 1. Animated build instructions. [View online] burning cigarettes (Liu et al., 2016), or filling chambers manually by emptying bags filled with vaporized cannabis (Manwell et al., 2014; Nelong et al., 2019). As these methods are inconsistent and are not compatible with modern vaping devices, others have begun to develop more sophisticated vapor exposure setups. The designs to date, however, are generally made for specific use cases and are expensive and complicated to construct. One such design used an atomizer to create vapor, which is pulled through a sealed chamber by an exhaust valve (Nguyen et al., 2016; Freels et al., 2020; Montanari et al., 2020). Unfortunately, the custom interface that triggers this device and other components are complex to construct and operate, and the system costs upwards of $\$ 20,000$.

Simpler designs have also been implemented. An e-cigarette exposure apparatus by Lefever et al. (2017) is most similar to OV in its low-cost and simple construction; however, similar to the previously described device, it makes use of an atomizer for vapor generation, therefore limiting its use to e-liquids. OV can be used with any vaporizer and has been used with both pod devices and cannabis flower vaporizers. Some custom systems have been designed to be compatible with both e-cigarettes and combustible cigarettes such as that created by Hage et al. (2017). While the design is similar to OV, it is more complicated, not truly open-source, and more expensive. Additionally, each chamber must be calibrated before every use and some of the design components are housed inside the chamber where they can potentially be

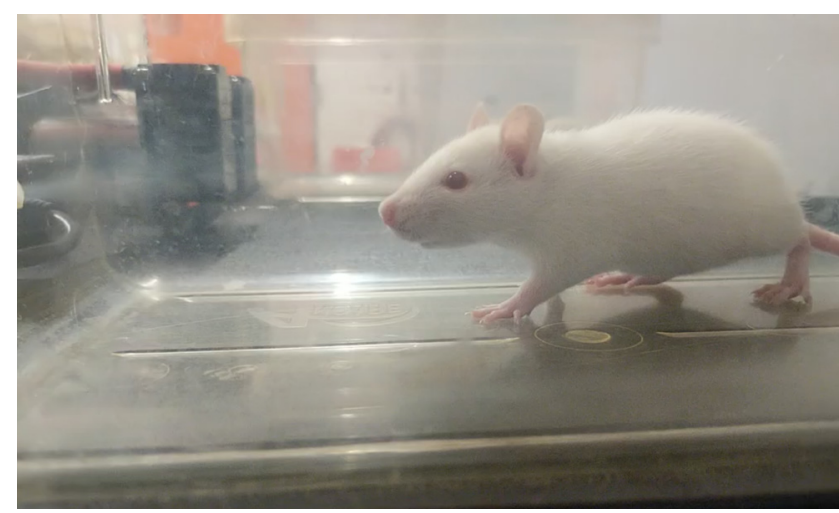

Movie 2. Video of device in operation. [View online] 
damaged by animals. All of OV's components are outside of the chambers, making it is impossible for the animals to harm themselves or damage the device. Another apparatus can customize the air-flow through the chamber, monitor the aerosol content, and control puff topography (Hilpert et al., 2019). While cheaper than other commercial technologies, the full apparatus (microcontroller, circuitry, non-open-source remote peristaltic pump, etc.) still costs approximately $\$ 1200$ more than OV.

One major benefit of using our system is that it gives labs the ability to use commercially available pods. This benefit is demonstrated in this study with plasma nicotine levels observed in our animals (after 2-8 min of vapor delivery) close to those observed in previous studies after 60 min of exposure at similar doses (Montanari et al., 2020). Importantly, the nicotine levels following the 8-min exposure duration are also similar to levels achieved by rats self-administering $0.5 \mathrm{mg} / \mathrm{ml}$ nicotine e-liquid vapor in a 60-min session (Smith et al., 2020), further supporting the rewarding nature of nicotine vapor (like our CPP findings) at the plasma levels reached in our study. To date, nearly all other studies testing the effects of vaporized nicotine have used laboratory grade nicotine dissolved in their vehicles. While this allows for the ability to adjust dosing, it does not capture the novel methods of increasing nicotine bioavailability used by e-cigarette manufacturers (especially in pod-designs like JUUL; Duell et al., 2019).

While it can be useful for many applications, our device has limitations. One limitation of our design, and others', is the lack of dosage measurement. The amount of nicotine e-liquid expended from the JUUL could be measured, however, a considerable amount of the vapor remains in the chamber without being inhaled. The rats may also react differently during the vapor administration, meaning some inhale more vapor than others, possibly leading to the high variability and the lack of statistically significant differences between doses in nicotine. Unfortunately, this device cannot alter the dosage based on breathing rate, nor can it account for age-dependent differences in tidal volume. However, plasma nicotine levels can be measured to assess the amount of nicotine absorbed by the rats as we have done in this study.

In conclusion, OV provides researchers with a low-cost and effective method of regulated e-cigarette vapor exposure for rodents. Our open-source design also allows for replication and customization. As we, and others, continue to improve methods of vapor exposure, important findings regarding the neurobiological effects of vaping can be revealed. OV allows for easy implementation of vapor exposure paradigms in a variety of experimental designs for the behavioral neuroscience, as well as the broader science, community.

\section{References}

Ahsan HM, de la Peña JBI, Botanas CJ, Kim HJ, Yu GY, Cheong JH (2014) Conditioned place preference and self-administration induced by nicotine in adolescent and adult rats. Biomol Ther (Seoul) 22:460-466.

Alasmari F, Crotty Alexander LE, Drummond CA, Sari Y (2018) A computerized exposure system for animal models to optimize nicotine delivery into the brain through inhalation of electronic cigarette vapors or cigarette smoke. Saudi Pharm J 26:622-628.

Belluzzi JD, Lee AG, Oliff HS, Leslie FM (2004) Age-dependent effects of nicotine on locomotor activity and conditioned place preference in rats. Psychopharmacology (Berl) 174:389-395.

Benowitz NL (1999) Nicotine addiction. Prim Care 26:611-631.

Brielmaier JM, McDonald CG, Smith RF (2007) Immediate and longterm behavioral effects of a single nicotine injection in adolescent and adult rats. Neurotoxicol Teratol 29:74-80.

Clarke PBS, Fibiger HC (1987) Apparent absence of nicotine-induced conditioned place preference in rats. Psychopharmacology (Berl) 92:84-88.

Craig EL, Zhao B, Cui JZ, Novalen M, Miksys S, Tyndale RF (2014) Nicotine pharmacokinetics in rats is altered as a function of age, impacting the interpretation of animal model data. Drug Metab Dispos 42:1447-1455.

Craver R (2019) Juul ends 2018 with 76 percent market share. Winston-Salem J Jan 8, 2019.

DiFranza JR, Rigotti NA, McNeill AD, Ockene JK, Savageau JA, St Cyr D, Coleman M (2000) Initial symptoms of nicotine dependence in adolescents. Tob Control 9:313-319.

Duell AK, Pankow JF, Peyton DH (2019) Nicotine in tobacco product aerosols: it's déjà vu all over again. Tob Control. Advance online publication. Retrieved. doi:10.1136/tobaccocontrol-2019-055275.

Freels TG, Baxter-Potter LN, Lugo JM, Glodosky NC, Wright HR, Baglot SL, Petrie GN, Yu Z, Clowers BH, Cuttler C, Fuchs RA, Hill MN, McLaughlin RJ (2020) Vaporized cannabis extracts have reinforcing properties and support conditioned drug-seeking behavior in rats. J Neurosci 40:1897-1908.

Hage AN, Krause W, Mathues A, Krasner L, Kasten S, Eliason JL, Ghosh A (2017) Comparing the effects of electronic cigarette vapor and cigarette smoke in a novel in vivo exposure system. $J$ Vis $\operatorname{Exp}(123): 55672$.

Hilpert M, Ilievski V, Coady M, Andrade-Gutierrez M, Yan B, Chillrud SN, Navas-Acien A, Kleiman NJ (2019) A custom-built low-cost chamber for exposing rodents to e-cigarette aerosol: practical considerations. Inhal Toxicol 31:399-408.

Huang J, Duan Z, Kwok J, Binns S, Vera LE, Kim Y, Szczypka G, Emery SL (2019) Vaping versus JUULing: how the extraordinary growth and marketing of JUUL transformed the US retail e-cigarette market. Tob Control 28:146-151.

Kavuluru R, Han S, Hahn EJ (2019) On the popularity of the USB flash drive-shaped electronic cigarette Juul. Tob Control 28:110-112.

Kong G, Bold KW, Morean ME, Bhatti H, Camenga DR, Jackson A, Krishnan-Sarin S (2019) Appeal of JUUL among adolescents. Drug Alcohol Depend 205:107691.

Kota D, Martin BR, Robinson SE, Damaj MI (2007) Nicotine dependence and reward differ between adolescent and adult male mice. $J$ Pharmacol Exp Ther 322:399-407.

Kota D, Martin BR, Damaj MI (2008) Age-dependent differences in nicotine reward and withdrawal in female mice. Psychopharmacology (Berl) 198:201-210.

Kota D, Robinson SE, Imad Damaj M (2009) Enhanced nicotine reward in adulthood after exposure to nicotine during early adolescence in mice. Biochem Pharmacol 78:873-879.

Leavens ELS, Stevens EM, Brett El, Leffingwell TR, Wagener TL (2019) JUUL in school: JUUL electronic cigarette use patterns, reasons for use, and social normative perceptions among college student ever users. Addict Behav 99:106047.

Lefever TW, Lee YOK, Kovach AL, Silinski MAR, Marusich JA, Thomas BF, Wiley JL (2017) Delivery of nicotine aerosol to mice via a modified electronic cigarette device. Drug Alcohol Depend 172:80-87.

Le Foll B, Goldberg SR (2005) Nicotine induces conditioned place preferences over a large range of doses in rats. Psychopharmacology (Berl) 178:481-492.

Lenoir M, Starosciak AK, Ledon J, Booth C, Zakharova E, Wade D, Vignoli B, Izenwasser S (2015) Sex differences in conditioned nicotine reward are age-specific. Pharmacol Biochem Behav 132:5662. 
Liu X, Wang Z, Li T, Ni L, Zeng R, Liu C (2016) A simplified device for exposing rats to cigarette smoke. Int J Clin Exp Med 9:1921319221.

Mantey DS, Cooper MR, Clendennen SL, Pasch KE, Perry CL (2016) $\mathrm{E}$-cigarette marketing exposure is associated with e-cigarette use among US youth. J Adolesc Health 58:686-690.

Manwell LA, Ford B, Matthews BA, Heipel H, Mallet PE (2014) A vapourized $\Delta 9$-tetrahydrocannabinol $(\Delta(9)$-THC) delivery system part II: comparison of behavioural effects of pulmonary versus parenteral cannabinoid exposure in rodents. J Pharmacol Toxicol Methods 70:112-119.

Matta SG, Balfour DJ, Benowitz NL, Boyd RT, Buccafusco JJ, Caggiula AR, Craig CR, Collins AC, Damaj MI, Donny EC, Gardiner PS, Grady SR, Heberlein U, Leonard SS, Levin ED, Lukas RJ, Markou A, Marks MJ, McCallum SE, Parameswaran N, et al. (2007) Guidelines on nicotine dose selection for in vivo research. Psychopharmacology (Berl) 190:269-319.

Montanari C, Kelley LK, Kerr TM, Cole M, Gilpin NW (2020) Nicotine e-cigarette vapor inhalation effects on nicotine and cotinine plasma levels and somatic withdrawal signs in adult male Wistar rats. Psychopharmacology (Berl) 237:613-625.

Nelong TF, Jenkins BW, Perreault ML, Khokhar JY (2019) Extended attenuation of corticostriatal power and coherence after acute exposure to vapourized $\Delta 9$-tetrahydrocannabinol in rats. Can $\mathrm{J}$ Addict 10:60-66.

Nguyen JD, Aarde SM, Vandewater SA, Grant Y, Stouffer DG, Parsons LH, Cole M, Taffe MA (2016) Inhaled delivery of $\Delta$ (9)-tetrahydrocannabinol (THC) to rats by e-cigarette vapor technology. Neuropharmacology 109:112-120.

Omaiye EE, McWhirter KJ, Luo W, Pankow JF, Talbot P (2019) Highnicotine electronic cigarette products: toxicity of JUUL fluids and aerosols correlates strongly with nicotine and some flavor chemical concentrations. Chem Res Toxicol 32:1058-1069.

Rao P, Liu J, Springer ML (2020) JUUL and combusted cigarettes comparably impair endothelial function. Tob Regul Sci 6:30-37.

Shoaib M, Stolerman IP, Kumar RC (1994) Nicotine-induced place preferences following prior nicotine exposure in rats. Psychopharmacology (Berl) 113:445-452.
Shram MJ, Lê AD (2010) Adolescent male Wistar rats are more responsive than adult rats to the conditioned rewarding effects of intravenously administered nicotine in the place conditioning procedure. Behav Brain Res 206:240-244.

Shram MJ, Funk D, Li Z, Lê AD (2006) Periadolescent and adult rats respond differently in tests measuring the rewarding and aversive effects of nicotine. Psychopharmacology (Berl) 186:201-208.

Smith LC, Kallupi M, Tieu L, Shankar K, Jaquish A, Barr J, Su Y, Velarde N, Sedighim S, Carrette LLG, Klodnicki M, Sun X, de Guglielmo G, George O (2020) Validation of a nicotine vapor selfadministration model in rats with relevance to electronic cigarette use. Neuropsychopharmacology.

Substance Abuse and Mental Health Services Administration (2013) Results from the 2012 National Survey on Drug Use and Health: summary of national findings. NSDUH Ser. H-46, HHS

Thorpe HHA, Hamidullah S, Jenkins BW, Khokhar JY (2020) Adolescent neurodevelopment and substance use: receptor expression and behavioral consequences. Pharmacol Ther 206:107431.

Torres OV, Tejeda HA, Natividad LA, O'Dell LE (2008) Enhanced vulnerability to the rewarding effects of nicotine during the adolescent period of development. Pharmacol Biochem Behav 90:658-663.

Torres OV, Natividad LA, Tejeda HA, Van Weelden SA, O'Dell LE (2009) Female rats display dose-dependent differences to the rewarding and aversive effects of nicotine in an age-, hormone-, and sex-dependent manner. Psychopharmacology (Berl) 206:303312.

Vastola BJ, Douglas LA, Varlinskaya EI, Spear LP (2002) Nicotine-induced conditioned place preference in adolescent and adult rats. Physiol Behav 77:107-114.

Wang TW, Gentzke AS, Creamer MR, Cullen KA, Holder-Hayes E, Sawdey MD, Anic GM, Portnoy DB, Hu S, Homa DM, Jamal A, Neff LJ (2019) Tobacco product use and associated factors among middle and high school students - United States, 2019. MMWR Surveill Summ 68:1-22.

Yararbas G, Keser A, Kanit L, Pogun S (2010) Nicotine-induced conditioned place preference in rats: sex differences and the role of mGluR5 receptors. Neuropharmacology 58:374-382. 